\title{
An Accelerated Iterative Cone Beam Computed Tomography Image Reconstruction Approach
}

\author{
Shimaa Abdulsalam Khazal ${ }^{1}$, Mohammed Hussein $\mathrm{Ali}^{2^{*}}$
}

\section{Authors affiliations: \\ 1) Electronic and \\ Communication Engineering, \\ Al-Nahrain University, \\ Baghdad-Iraq. \\ shimaa9191@gmail.com \\ 2*) Electronic and \\ Communication Engineering, \\ Al-Nahrain University, \\ Baghdad-Iraq. \\ malhayani@gmail.com}

\section{Paper History:}

Received: $22^{\text {th }}$ Sep. 2019

Revised: $25^{\text {th }}$ Oct. 2019

Accepted: $17^{\text {th }}$ Nov. 2019

\begin{abstract}
Cone-beam computed tomography (CBCT) is an indispensable method that reconstructs three dimensional (3D) images. CBCT employs a mathematical technique of reconstruction, which reveals the anatomy of the patient's body through the measurements of projections. The mathematical techniques employed in the reconstruction process are classified as; analytical, and iterative. The iterative reconstruction methods have been proven to be superior over the analytical methods, but due to their prolonged reconstruction time those methods are excluded from routine use in clinical applications. The aim of this research is to accelerate the iterative methods by performing the reconstruction process using a graphical processing unit (GPU). This method is tested on two iterative-reconstruction algorithms (IR), the algebraic reconstruction technique (ART), and the multiplicative algebraic reconstruction technique (MART). The results are compared against the traditional ART, and MART. A 3D test head phantom image is used in this research to demonstrate results of the proposed method on the reconstruction algorithms. The simulation results are executed using MATLAB (version R2018b) programming language and computer system with the following specifications: CPU core i7 $(2.40 \mathrm{GHz})$ for the processing, with a NIVDIA GEFORCE GPU. Experimental results indicate, that this method reduces the reconstruction time for the iterative algorithms.
\end{abstract}

Keywords: Cone-Beam Computed Tomography, ART, MART, GPU, SheppLogan, 3D, Iterative

$$
\begin{aligned}
& \text { تسريع الطرق التكرارية لاعادة بناء الصورة التومغرافية في الشعاع الخخروطي } \\
& \text { شياء عبد السلام خزعل ، محمد حسين علي } \\
& \text { التصوير المقطعي المحوسب للأشعة الخروطية (CBCT) هو طريقة تصوير قيمة للغاية تعيد بناء الصور ثلاثية }
\end{aligned}
$$

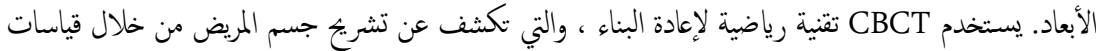

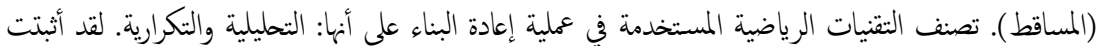

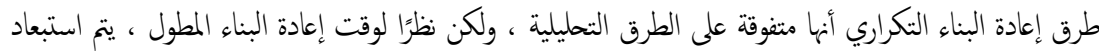

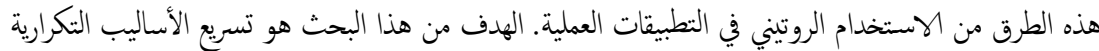

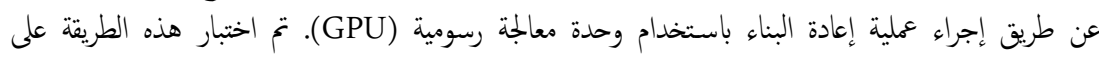

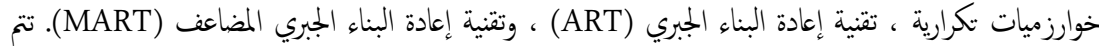

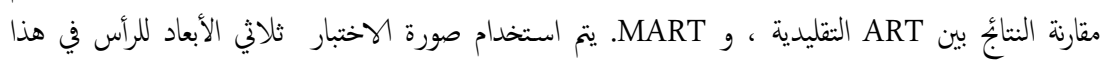

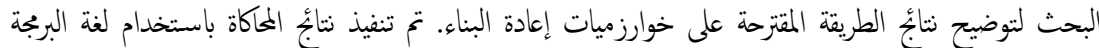

$$
\begin{aligned}
& \text { 2.40 (الإصدار RATLAB }
\end{aligned}
$$

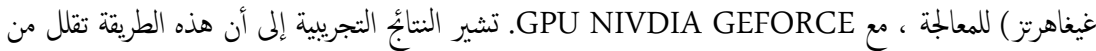

$$
\begin{aligned}
& \text { وقت إعادة البناء للخوارزميات التكرارية. }
\end{aligned}
$$




\section{Introduction}

Computed tomography (CT) was first introduced in the early 1970s. It was formerly known as computed axial tomography commonly known as (CAT) scanning; the word computed was incorporated in the description to signify the key role of the computer in the improvement of this technology [1]. Traditional X-ray radiography produces a two-dimensional (2D) figure from the three-dimensional (3D) body. CT has revolutionized radiology by acquiring thin slices of the scanned object in a non-invasive manner via an X-ray beam rotating around the object, thus constructing sliced images of every cross-section [2].

CBCT is considered to be an efficient, prompt, and safer modified-version of the traditional CT scanner, since the scanner size, the dose of radiation and the scanning time are all minimized significantly. The first generation of CBCT scanners was first introduced in 1982. In CBCT a cone-shaped X-ray beam source is utilized, along with a detector unit that is formed as a screen as shown in Fig. 1 [3]. These CT systems are capable of reconstructing 3D images. The scanning process is completed in a single $\left(360^{\circ}\right)$ degrees rotation, in which the radiation source and the opposite side detector are rotated about the scanned object [4].

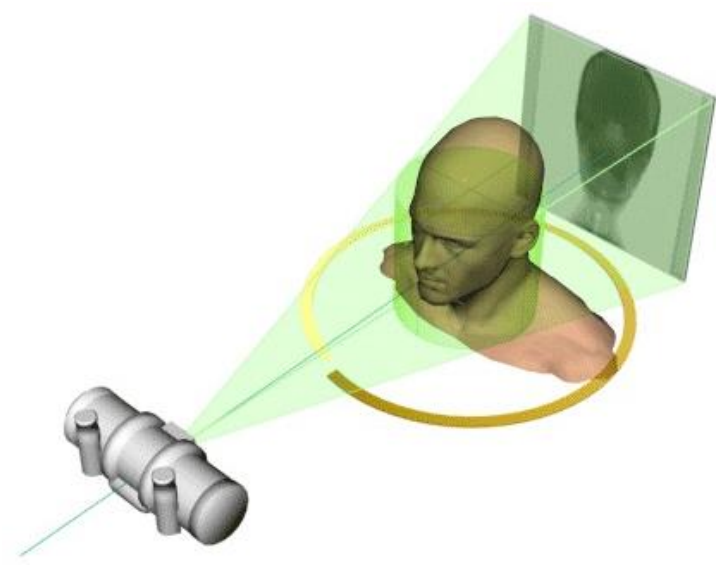

Figure (1): CBCT scanner [3].

The foremost challenge in CT imaging is the reconstruction of images from the acquired set of projections [5]. There are two major approaches to CT image reconstruction; analytical and iterative. The analytical approach is commonly defined by the filtered back projection algorithm (FBP). This approach filters the acquired set of projections then back projects the filtered projections onto the reconstruction grid, this algorithm produces 2D images. However, the iterative reconstruction (IR) approach produces $2 \mathrm{D}$ and $3 \mathrm{D}$ images, it branches into a wide variety of algorithms, for example; the algebraic reconstruction technique (ART), the multiplicative algebraic reconstruction technique (MART), the simultaneous algebraic reconstruction technique (SART), the simultaneous iterative reconstruction technique (SIRT), and many more. These algorithms start with an initial guess of the scanned object. This initial guess is then updated and corrected many times according to the acquired projection data, and depending on the number of iterations as well as on the type of the utilized algorithm, which makes the iterative approach more demanding in the computational power because of the large number of calculations it requires [6]. MarieLouise Aurumskjöld [7] evaluated and optimized IR methods in CT regarding image quality and radiation dose. The author included a combination of studies on phantoms and patients, and showed that IR algorithms have different strengths and weaknesses, but the important conclusion in this work is that all vendors' IR algorithms improve image quality. Dejian Qiu, and Euclid Seeram [8] purposed a review to ascertain if there is a general agreement that IR algorithms reduce radiation dose and improve image quality in $\mathrm{CT}$ in comparison with the analytical reconstruction algorithms. The authors found that the use of IR algorithms reduces objective image noise, and sustains spatial resolution, even when the dose is reduced. Fang $\mathrm{Xu}$, and Klaus Mueller [9] illustrated how graphical processing units (GPUs) could be utilized to perform 3D analytical and iterative image reconstruction algorithms. The techniques presented in this paper established that the recent revolution in PC graphics board technology has immense potential for CT. Tessa Van Hemelryck et al. [10] utilized three eminent iterative techniques ART, SART and SIRT. The algorithms implementations and calculations were carried out in MATLAB and on a GPU. Xing Zhao, Jing-jing Hu, and Peng Zhang [11], explored and executed a GPU based 3D CBCT image reconstruction algorithm for a large volume of data. Claudia de Molina et al. [12] proposed a GPU-accelerated iterative reconstruction for limited-data in CBCT systems. Tomás Antonio Valencia Pérez et al. [13] employed a GPU to reconstruct the Shepp-Logan phantom image. The results showed that, an acceptable image reconstruction was completed faster than the traditional reconstruction methods. Ander Biguri [14] utilized the GPU in the reconstruction of $2 \mathrm{D} C \mathrm{CT}$ images. $\mathrm{Yi} \mathrm{Du}$ et al. [15] proposed a GPU acceleration method for calculating voxel-driven forward projections in cone-beam CT. The experimental results demonstrate its effectiveness and efficiency in handling the inter-thread interference problem.

In this research; the $3 \mathrm{D}$ reconstruction process of two iterative algorithms ART, and MART is optimized by utilizing a GPU unit, the obtained results are compared with the traditional algorithms before applying the acceleration approach.

\section{Iterative CBCT image Reconstruction}

The iterative reconstruction process is considered as a "closed loop" approach in which every loop is called an iteration, which compares the simulated projections with the calculated projections, and then back-projections the recorded data inconsistencies between them. The data inconsistencies are employed to correct the initial guess image [14]. Iterative methods improve the quality of the image when it is 
not feasible or when the number of acquired projections is diminutive. Also, this approach accomplishes an improved image when the projections are not distributed evenly around the scan trajectory [16].

The iterative reconstruction algorithms are employed to provide a solution for a system of linear equations, or a minimization to an objective function. Elucidating a system of linear equations is achieved through the discretization of the image into a grid of $2 \mathrm{D}$ pixels or $3 \mathrm{D}$ voxels (volumetric pixels) for $3 \mathrm{D}$ images, as described in Fig. 2 [5], [6].

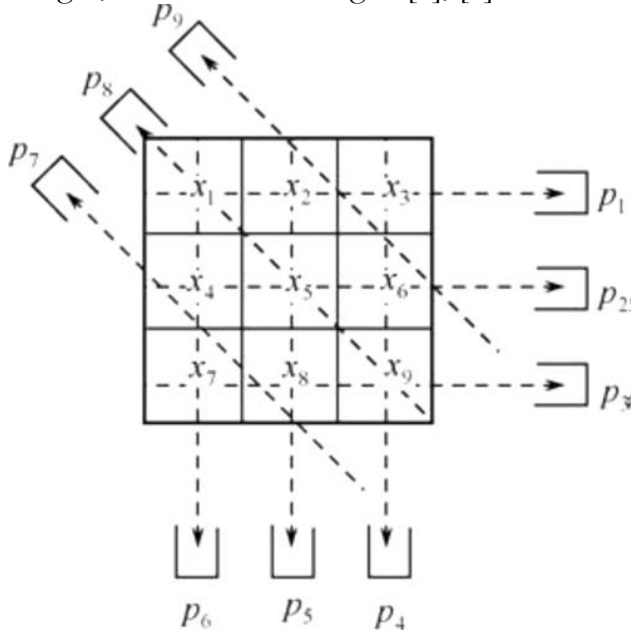

Figure (2): A linear equations system [6].

This figure $(x)$ represents the image pixels, and the projections are $(p)$, they could be related via linear equations as shown by Eq. (1) [6]:

$x_{1}+x_{2}+x_{3}=p_{1}$

$x_{4}+x_{5}+x_{6}=p_{2}$

$x_{7}+x_{8}+x_{9}=p_{3}$

$x_{3}+x_{6}+x_{9}=p_{4}$

$x_{2}+x_{5}+x_{8}=p_{5}$ $2(\sqrt{2}-1) x_{4}+(2-\sqrt{2}) x_{7}+2(\sqrt{2}-1) x_{8}=p_{7}$

$(\sqrt{2}) x_{1}+(\sqrt{2}) x_{5}+(\sqrt{2}) x_{9}=p_{8}$

$2(\sqrt{2}-1) x_{2}+(2-\sqrt{2}) x_{3}+2(\sqrt{2}-1) x_{6}=p_{9}$

This system could be re-written in the matrix form as demonstrated by Eq. (2) [6]:

$$
A X=P \quad \ldots(2)
$$

Where $X=\left[x_{1}, x_{2}, \ldots, x_{9}\right]^{T}, P=\left[p_{1}, p_{2}, \ldots, p_{9}\right]^{T}$, and $(A)$ is the coefficient matrix of the system. If the inverse matrix $\left(A^{-1}\right)$ of $(A)$ exists, the reconstructed image is given by Eq. (3) [6].

$$
X=A^{-1} P
$$

The significant size of the matrix $(A)$ prevents its storage in a computer system, so this matrix is created in a row by row manner only. Any iterative method that modifies $(A)$ couldn't be utilized. Instead methods that employ matrix $(A)$ and its transposed matrix $\left(A^{-1}\right)$ are used [6]. Mathematically this means that all iterative reconstruction (IR) algorithms evaluate the reconstruction process starting with the first estimate of the image $(x)$, which is assumed to be zeros, this value is updated in a manner that relies on the IR algorithm employed.

The conjecture of IR algorithms is founded on six important stages that are shown in Fig. 3. Those steps follow the CT projection measurements; the first step is to generate an initial guess image, then the simulated projections are generated. The next step compares the simulated projection data with the projections measured from the CT scanner. If any discrepancies occur, the estimated image is corrected based on update equation of the utilized IR algorithm. This process of correcting the image is replicated, until the satisfaction of a condition that was predefined by the algorithm. The final image is generated when that condition is gratified [17].

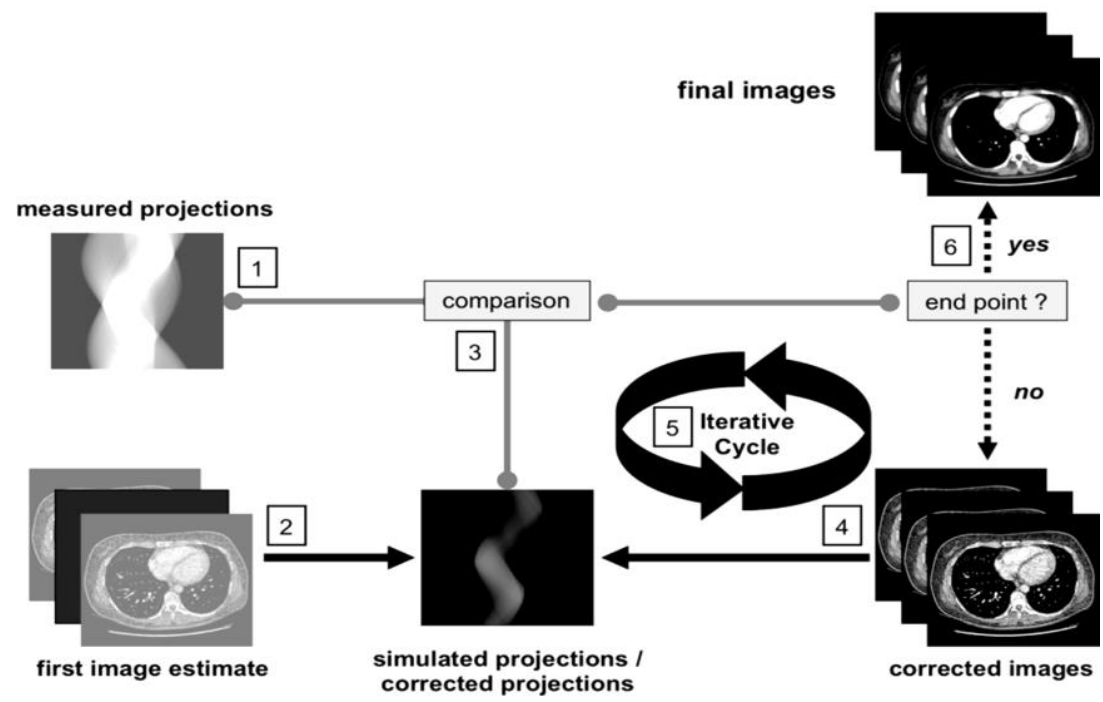

Figure (3): The basic steps of IR algorithms [18].

\section{A. ART Algorithm}

The principle of the ART algorithm (also known as the Kaczmarz algorithm) is to correct the estimated image, and satisfy one equation at a time [6]. Historically, the ART algorithm was the first algorithm applied in CT. The execution of this 
algorithm is processed one projection ray at a time, the reconstructed image is modified after each ray is measured.

ART algorithm function by using the information of only one beam sum (i.e. projection ray) at a time as shown in Fig. 4. This algorithm begins with an estimate of the scanned object. This estimate is modified by considering the sum of the voxels along a straight ray, this sum is then compared with the measured projections for that ray. The difference between the calculated projections obtained from the image estimate and the projections measured from the passing X-ray beam, is used to update the initial image. Therefore, ART is occasionally titled the rayby-ray reconstruction method [19].

Figure 4 shows a projection beam $\left(P_{i}\right)$ of width $(\Delta \xi)$, passing through the tissue. The pixel size is given by $(b)$. The weight $\left(a_{i j}\right)$ is thus determined by Eq. (4) [19].

$$
\begin{array}{r}
\text { aij }=(\text { illuminated area of pixel } j \text { by ray } I) \\
/(\text { total area of pixel } j) \ldots(4)
\end{array}
$$

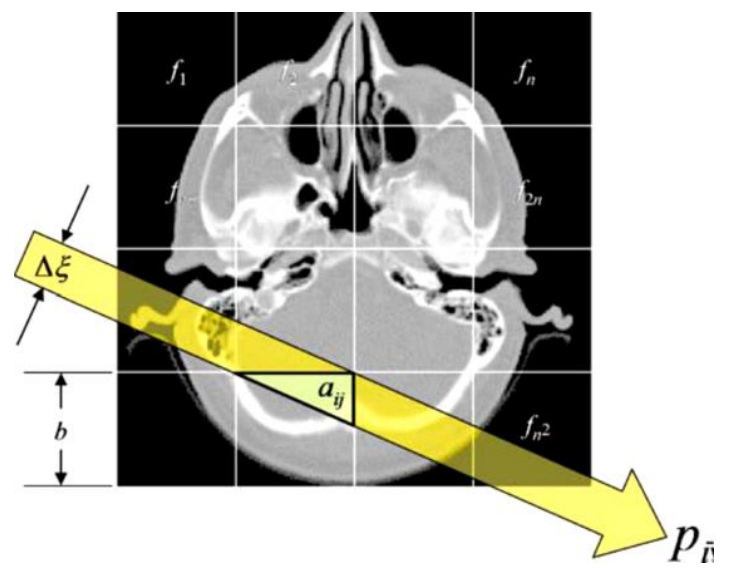

Figure (4): Demonstration of a single beam sum passing through an object [19].

The method of image reconstruction using the ART algorithm is sum up in the following steps [19]:

1. An initial guess image $\left(X^{\text {current }}\right)$ is suggested.

2. The initial guess image is corrected by employing Eq. (5) [6]:

$X^{\text {next }}=X^{\text {current }}-\lambda \frac{A_{i} X^{\text {current }}-p_{i}}{\left\|A_{i}\right\|^{2}} A_{i}{ }^{T}$.

In this equation $(\lambda)$ is the relaxation parameter, which is usually chosen to be $(1),\left(p_{i}\right)$ are the measured projections, and $\left(A_{i}\right)$ is the contribution factor of the matrix along the $i$ th ray. $\left\|A_{i}\right\|^{2}=$ $\sum_{j} a_{i j}{ }^{2}$ Is the squared sum of the "contribution factors" for the $i$ th ray.

3. The previous steps are repeated starting from the second step, depending on the number of iterations.

\section{B. MART Algorithm}

MART algorithm is an iterative reconstruction technique. This algorithm is capable of constructing a 3D image using a large number of linear equations. MART algorithm is similar to the ART algorithm, but it differs in the correction stage which is multiplicative. This means that it is implemented using an initial guess image with any value other than zero. The MART technique includes a multiplicative update to the voxel intensity generated from the previous iteration, based on the fraction of the measured projections, and the calculated projections.

The distinctive quality of the MART algorithm is its non-negativity. If the estimated image does not enclose pixels or voxels with a negative value, the reconstructed image will never be negative [6]. Many studies reveal that this reconstruction approach is faster, more flexible, and more accurate than the ART algorithm, especially when the number of projections is limited [20]. MART is considered to be a row-action algorithm, as expressed by Eq. (6) [21].

$$
X^{\text {next }}=X^{\text {current }} *\left(\frac{p_{i}}{A_{i} X^{\text {current }}}\right)^{\lambda} \text {. }
$$

The parameters in this equation are similar to Eq. (5) of the ART algorithm. The initial guess image must start with any value other than (0), if the measured projection is equal to zero, then all of the voxels that were measured by the rays will be set to zero, indicating that none of them contribute to the ray sum.

\section{The Graphical Processing Unit}

GPUs are categorized as a vastly multi-thread, multi core parallel processor with a remarkable floating-point processing ability. GPUs are employed in iterative reconstruction processing because the step with the most time exhaustion in these algorithms, is the multiplication of the matrix-vector. In GPUs, the vast number of pixels and detector data could be processed in a parallel manner, which makes their processing much faster than in CPUs. The employment of GPUs in medical image processing has been significantly augmented in the recent years because, GPUs have become more dominant. Medical imaging, often utilize techniques that include repetitive computations performed on substantial multi-dimensional sets of data that are well suited for the parallel processing capabilities of GPUs. These imaging techniques use graphics cards that utilizes the processing characteristics of the CPU and the GPU. The CPU is used to execute the sequential portion of the algorithm, and the most "expensive" portion of the calculation is carried out on the GPU as shown in Fig. 5 [13] and [21]. 


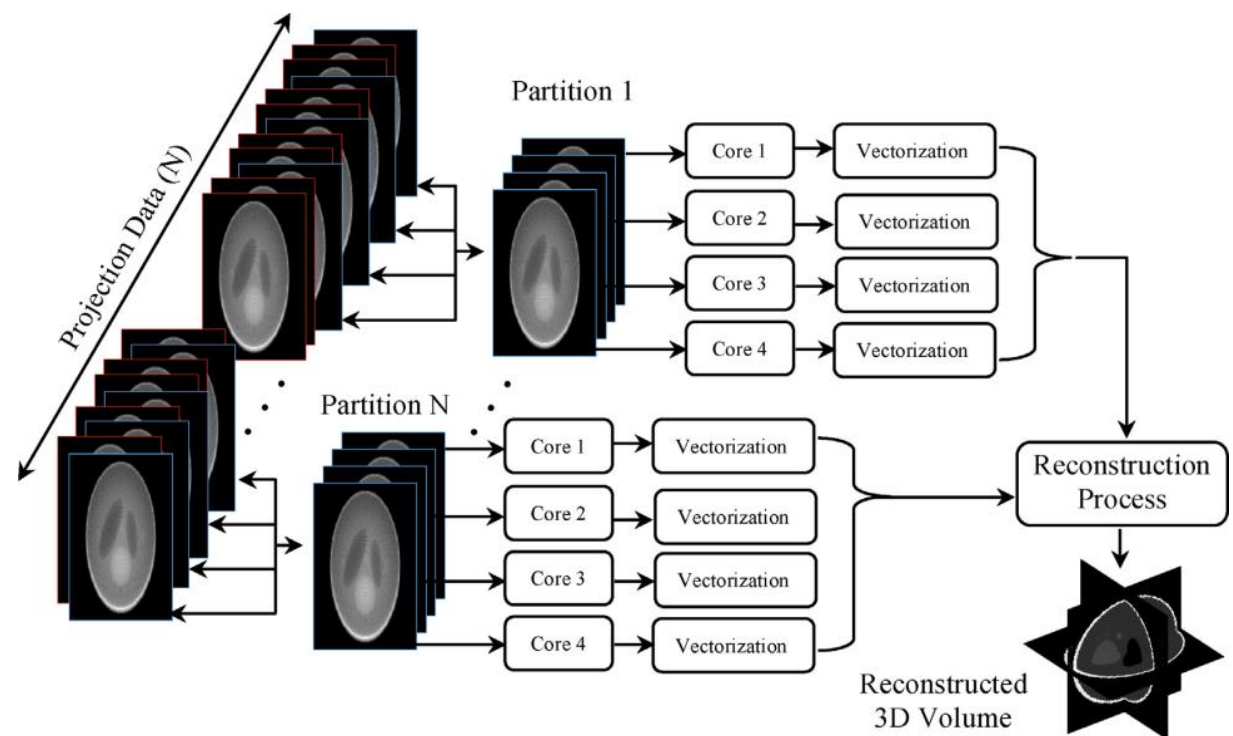

Figure (5): Parallelization of the projection data for 3D image reconstruction [21].

This figure shows that GPUs are capable of processing large sets of pixels, and projection data in a parallel manner. This parallel process is an operational mode in which a procedure is divided into portions that are simultaneously executed on different processors connected to the same computer, thus processing them much faster than CPUs. The algorithm will execute prompter because it is employing the accelerated performance of the GPU to the process [13].

\section{Computer Simulation of the Accelerated Reconstruction Algorithms}

In this research the reconstruction time of two types of iterative reconstruction algorithms: ART, and MART, is reduced by; executing these algorithms using a NIVIDIA GEFORCE GPU. This work is simulated using MATLAB. The following steps are employed to implement this work:

1. A 3D shepp logan head phantom which mimics all the possible interactions between brain tissues as close to the human body as possible. So, it is suitable for the simulation of the reconstruction process of a 3D CBCT scanner, is presented in Fig. 6 [22]. This phantom is generated to be used as an input test image to represent all the possible cases that could occur in the CBCT imaging of a human head.

2. Projections of the $3 \mathrm{D}$ segmented image are measured. Using a GPU to reduce the projection measuring time.

3. An initial guess image is generated, to be used for the iterative reconstruction process. The size of the generated image is the same size as the input test image.

4. The relaxation parameter $(\lambda)$ is specified, and the number of iterations for the iterative reconstruction process.

5. The calculated projections for the initial guess image are stimulated.

6. Iterative reconstruction processes of the ART, and MART algorithms are performed separately. Those algorithms start by comparing the calculated and the measured projections to obtain the correction, which is then used to update the initial guess image.

7. The process of iterative reconstruction will update the image of each algorithm depending on the number of iterations specified by step (5).

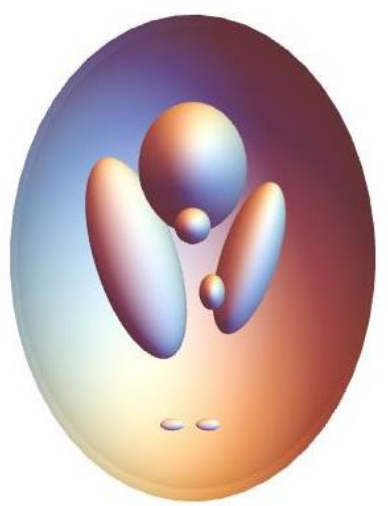

Figure (6): 3D shepp-logan head phantom [22].

\section{Simulation Results}

The prolonged reconstruction time of the iterative algorithms is one of the major drawbacks that prohibits the application of this approach in medical image reconstruction despite its greater advantages over the analytical approach. The results of the simulation of the accelerated algorithms are executed using MATLAB (version R2018b) programming language and computer system with the following specifications: CPU core i7 $(2.40 \mathrm{GHz})$ for the processing, with a NIVDIA GEFORCE graphical processing unit (GPU).

The results of the simulation, employed the proposed 3D shepp-logan head phantom, to test and carry out the simulated algorithms. This phantom is generated using a user defined matrix, the matrix rows signify ellipsoids in the image. The matrix is used to create a phantom that consists of (10) columns, each containing a special parameter for the ellipsoids. Fig. 7 shows three views of a (256 x 256) $3 \mathrm{D}$ shepp-logan head phantom, the middle slice (slice number 128) is shown. 


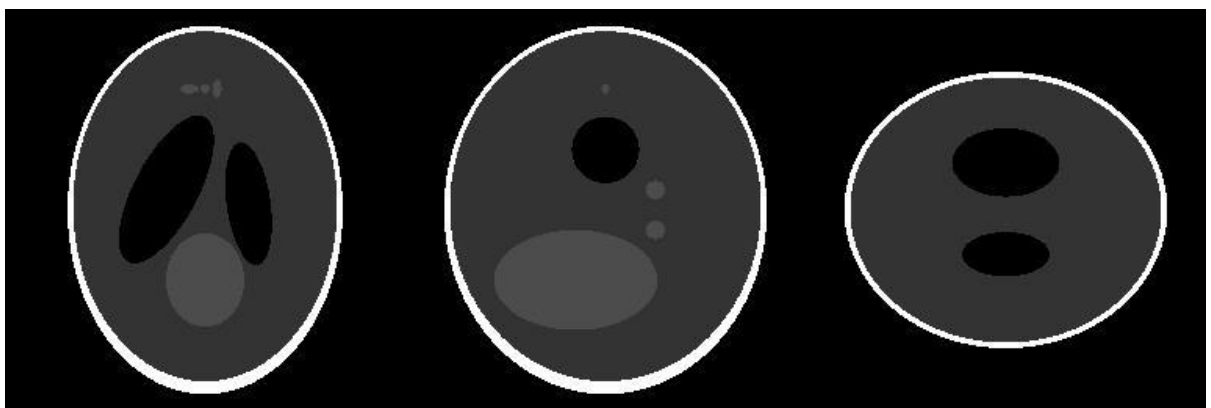

(A)

(B)

(C)

Figure (7): 3D shepp-logan phantom (A) Axial view, (B) Coronal view, and (C) Sagittal view.

Illustration of the three views in Fig.7, represents a slice taken from three different views, as shown in Fig.8. This figure shows the slice planes of the phantom along each axis $(x, y$, and $z)$.

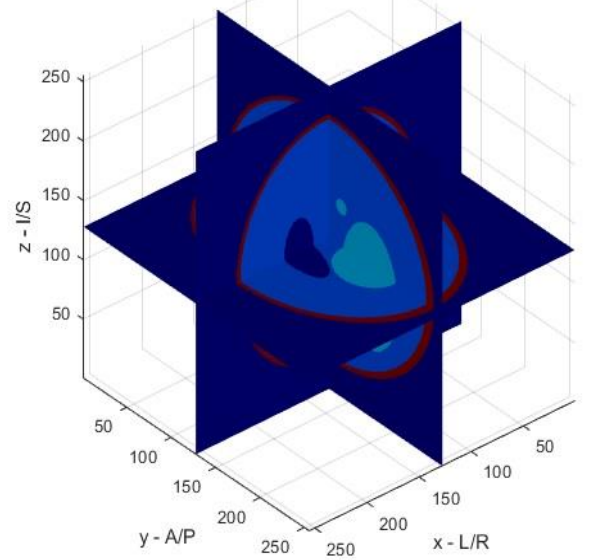

Figure (8): The plane slices of the $3 \mathrm{D}$ head phantom.

The simulation process of the ART algorithm is based on the iteration loop that begins with the first iteration, and ends when the chosen number of iterations which is (300) is completed. This number is chosen because it generates an image with a good quality while sustaining an acceptable reconstruction time. Choosing a value higher than (300) does not alter the image quality that much, but it will have an effect on the reconstruction time. Each iteration will update the initial guess image according to Eq. (5). The simulation of this equation is accelerated by utilizing the GPU. The reconstructed phantom image using ART is demonstrated in Fig. 9. The reconstruction time yielded from accelerating this algorithm is demonstrated in table 1 .

Table (1): Accelerated ART algorithm reconstruction time

\begin{tabular}{|c|c|}
\hline Algorithm & Time (sec.) \\
\hline ART & 113.892049 \\
\hline Accelerated ART & 95.548244 \\
\hline
\end{tabular}

This table shows that our approach of utilizing a GPU in the reconstruction process has noticeably accelerated the process time of the ART algorithm.

The reconstruction time of the MART algorithm is also accelerated using a GPU, each iteration updates the initial guess image in a multiplicative manner as shown by Eq. (6).

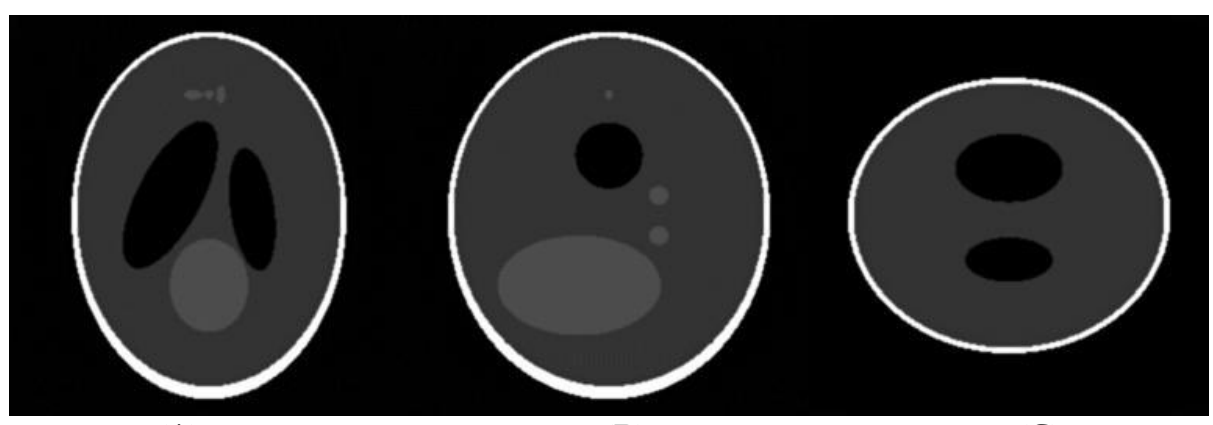

(A)

(C)

Figure (9): The reconstructed phantom image using the accelerated ART algorithm (A) Axial view, (B) Coronal view, and (C) Sagittal view.

Simulating the accelerated MART algorithm yielded a reconstruction time that is shown in table 2 . The reconstructed phantom image of the accelerated MART algorithm simulation is shown in Fig. 10.
Table (2): The reconstruction time of the accelerated MART algorithm

\begin{tabular}{|c|c|}
\hline Algorithm & Time \\
\hline MART & 90.323982 \\
\hline Accelerated MART & 76.425285 \\
\hline
\end{tabular}


The results in table 2 shows that this acceleration approach reduced the reconstruction time of the MART algorithm as well.

\section{Conclusions}

The proposed method performed in this research could be employed in the reconstruction of $3 \mathrm{D}$ CBCT images. This method is utilized to aid the reconstruction process of iterative algorithms by reducing their reconstruction time. The main conclusions that could be drawn from the implementation of the suggested method that is founded on the use of a GPU, when employed to the iterative, ART, and MART algorithms are surmised as follows:

1. Simulating the proposed acceleration method on the iterative ART algorithm reduced the reconstruction time by $(17.517 \%)$ in comparison to the traditional ART algorithm.

2. Accelerating the MART algorithm by using the suggested technique reduced the reconstruction time by $(16.6702 \%)$.

3. The MART algorithm exhibited that it was faster than the, ART algorithm.

This approach could be further supported by testing it on another iterative reconstruction algorithm. The quest for faster reconstruction of iterative CBCT images could be further improved by suggesting alternative approaches such as reducing the number of the reconstructed pixels, or by employing an analytical and iterative reconstruction process which is known as a hybrid algorithm to further reduce the reconstruction time.

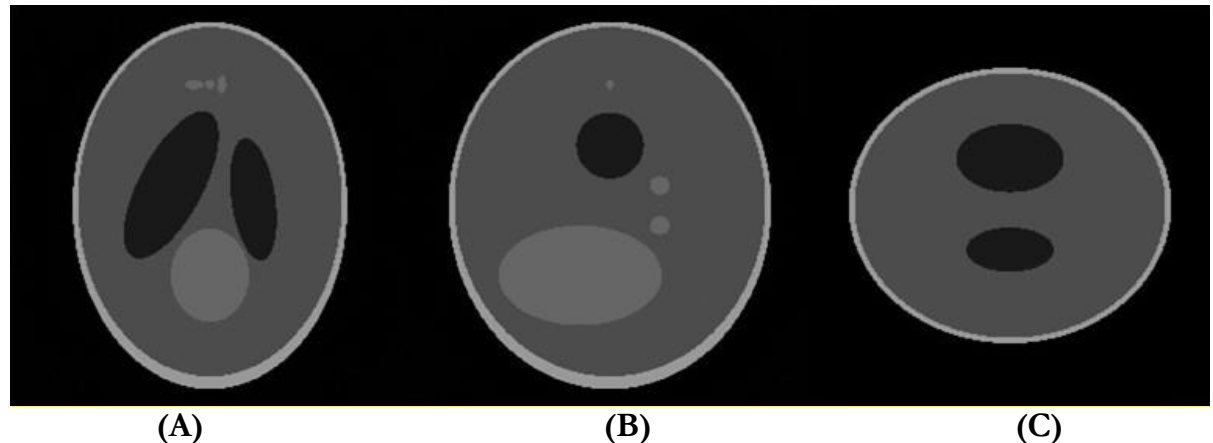

Figure (10): The reconstructed phantom image using the traditional MART algorithm (A) Axial view, (B) Coronal view, and (C) Sagittal view.

\section{References}

[1] P. Allisy-Robets and J. Williams, "Farr's physics for medical imaging", 2nd ed. Edinburgh: Elsevier, 2008.

[2] L. Romans, "Computed tomography for technologists". Philadelphia [etc.]: Wollters Kluwer Health/Lippincott Williams \& Wilkins, 2011.

[3] "Exxim computing corporation. Conventional Cone-Beam Setup", Exxim-cc.com, 2019. [Online]. Available: https://www.exximcc.com/conventional_cone_beam_setup.html. [Accessed: 26- Apr- 2019].

[4] P. Caruso, E. Silvestri and L. Sconfienza, "Cone beam CT and 3D imaging". Italy: Springer, 2014.

[5] A. Kak and M. Slaney, "Principles of computerized tomographic imaging". Philadelphia, Pa: IEEE, 1999.

[6] G. Zeng, "Medical Image Reconstruction”. Berlin, Heidelberg: Springer, 2010.

[7] M. Aurumskjöld, "Optimisation of image quality and radiation dose in computed tomography using iterative image reconstruction", MSc, Lund University, 2017.

[8] D. Qiu and E. Seeram, "Does Iterative Reconstruction Improve Image Quality and Reduce Dose in Computed Tomography?", Radiology - Open Journal, vol. 1, no. 2, pp. 4254, 2016. Available: 10.17140/roj-1-108.
[9] F. Xu and K. Mueller, “Accelerating popular tomographic reconstruction algorithms on commodity PC graphics hardware", IEEE Transactions on Nuclear Science, vol. 52, no. 3, pp. 654-663, $2005 . \quad$ Available: $10.1109 /$ tns.2005.851398.

[10] T. Van Hemelryck, S. Wuyts, M. Goossens, J. Batenburg Kees and J. Sijbers, "Iterative Reconstruction Algorithms The implementation of iterative reconstruction algorithms in MATLAB", 2007.

[11] X. Zhao, J. Hu and P. Zhang, "GPU-Based 3D Cone-Beam CT Image Reconstruction for Large Data Volume", International Journal of Biomedical Imaging, vol. 2009, pp. 1-8, 2009. Available: 10.1155/2009/149079.

[12] C. de Molina, E. Serrano, J. Garcia-Blas, J. Carretero, M. Desco and M. Abella, "GPUaccelerated iterative reconstruction for limiteddata tomography in CBCT systems", BMC Bioinformatics, vol. 19, no. 1, 2018. Available: 10.1186/s12859-018-2169-3

[13] T. Valencia Pérez, J. Hernández López, E. Moreno Barbosa, M. Martínez Hernández, G. Tejeda Muñoz and B. de Celis Alonso, "Parallel approach to tomographic reconstruction algorithm using a Nvidia GPU”, AIP Conference Proceedings, vol. 2090, no. 1, 2019. Available: $10.1063 / 1.5095930$.

[14] A. Biguri, "Iterative Reconstruction and Motion compensation in Computed Tomography on GPUs", MSc. University of Bath, 2017. 
[15] Y. Du, G. Yu, X. Xiang and X. Wang, "GPU accelerated voxel-driven forward projection for iterative reconstruction of cone-beam CT", BioMedical Engineering OnLine, vol. 16, no. 1, 2017. Available: 10.1186/s12938-016-0293-8.

[16] H. Scherl, "Evaluation of State-of-the-Art Hardware Architectures for Fast Cone-Beam CT Reconstruction”, 1st ed. Germany: Vieweg+Teubner Verlag, 2011.

[17] L. Geyer et al., "State of the Art: Iterative CT Reconstruction Techniques", Radiology, vol. 276, no. 2, pp. 339-357, 2015. Available: 10.1148/radiol.2015132766.

[18] M. Willemink and P. Noël, "The evolution of image reconstruction for CT from filtered back projection to artificial intelligence", European Radiology, vol. 29, no. 5, pp. 2185-2195, 2018. Available: $10.1007 / \mathrm{s} 00330-018-5810-7$.

[19] T. Buzug, "Introduction to Computed Tomography". Dordrecht: Springer, 2008.

[20] E. Oliveira, S. Melo, C. Dantas, D. Vasconcelos and L. Cadiz, "Comparison Among Tomographic Reconstruction Algorithms With A Limited Data", in International Nuclear Atlantic Conference, Belo Horizonte,MG,Brazil, 2011.

[21] M. Al-masni, M. Al-antari, M. Metwally, Y. Kadah, S. Han and T. Kim, "A rapid algebraic 3D volume image reconstruction technique for cone beam computed tomography", Biocybernetics and Biomedical Engineering, vol. 37, no. 4, pp. 619-629, 2017. Available: 10.1016/j.bbe.2017.07.001.

[22] "2D and 3D Shepp-Logan Phantom in the Fourier and Image Domains - HI-SPEED Software Packets", Sites.google.com, 2010. [Online]. Available: https://sites.google.com/site/hispeedpackets/ Home/shepplogan. [Accessed: 22- Sep- 2019].

[23] N. H. Fallooh Al-anbari and M. H. Ali AlHayani, "Design and Construction ThreeDimensional Head Phantom Test Image for the Algorithms of 3D Image Reconstruction", Journal of Emerging Trends in Computing and Information Sciences, vol. 6, no. 2, 2015.

[24] N. H. Fallooh Al-anbari and M. H. Ali AlHayani, "Evaluation Performance of Iterative Algorithms for 3D Image Reconstruction in Cone Beam Geometry", Al-Nahrain jounal of engineering sciences, vol. 20, no. 1, pp. 149-157, 2017. 